\title{
STRONG MARTINGALE CONVERGENCE OF GENERALIZED CONDITIONAL EXPECTATIONS ON VON NEUMANN ALGEBRAS
}

BY

\author{
FUMIO HIAI AND MAKOTO TSUKADA
}

\begin{abstract}
Accardi and Cecchini generalized the concept of conditional expectations on von Neumann algebras. In this paper we give some conditions for strong convergence of increasing or decreasing martingales of Accardi and Cecchini's conditional expectations.
\end{abstract}

Introduction. The study of conditional expectations and martingale convergence theorems in the operator algebraic framework was initiated by Umegaki's earlier works $[14,15]$. Since then, the martingale convergence theorems on von Neumann algebras have been developed by several authors. For example, the almost sure type martingale convergence was obtained by Lance [9] and Dang-Ngoc [4]. The strong martingale convergence was completed in [13] by one of the authors.

The conditional expectation of a von Neumann algebra $M$ onto a von Neumann subalgebra $N$ does not generally exist given a faithful normal state (or semifinite weight) $\varphi$ on $M$. Indeed its existence is equivalent to the invariance of $N$ under the modular automorphism group associated with $\varphi$ (see [12]). To recover this drawback of noncommutative conditional expectations, Accardi and Cecchini [1] generalized the concept of conditional expectations on von Neumann algebras by using the Tomita-Takesaki theory [11]. Accardi and Cecchini's conditional expectation $\varepsilon$ : $M \rightarrow N$ with respect to $\varphi$ always exists but is not necessarily a projection onto $N$ and lacks a useful property that $\varepsilon(a x b)=a \varepsilon(x) b$ for $a, b \in N$ and $x \in M$.

Araki [2] established the convergence of modular operators and modular conjugations given an increasing net of von Neumann subalgebras and a faithful normal state. By extending the arguments in [2], we have obtained in [8] the martingale type convergence of modular automorphism groups under an increasing or decreasing net of von Neumann subalgebras and a faithful normal semifinite weight. On the same lines, we investigate in this paper the strong convergence of martingales of Accardi and Cecchini's conditional expectations (called here generalized conditional expectations).

In $\S 1$ of this paper we state the definition of the generalized conditional expectation with respect to a faithful normal semifinite weight and give easy technical

Received by the editors March 15, 1983 and, in revised form, May 22, 1983.

1980 Mathematics Subject Classification. Primary 46L10.

Key words and phrases. Conditional expectation, martingale, Tomita-Takesaki theory, faithful normal semifinite weight, nonabelian $K$-flow.

1984 American Mathematical Society $0002-9947 / 84 \$ 1.00+\$ .25$ per page 
lemmas. In $\$ 2$ we establish necessary and sufficient conditions for the strong convergence of an increasing martingale of generalized conditional expectations. In $\$ 3$ we show the strong convergence of a decreasing martingale of generalized conditional expectations with suitable assumptions. Finally we have an application to some convergence properties for nonabelian $K$-flows.

The authors would like to express their gratitude to Professor H. Umegaki for his advice and constant encouragement.

1. Preliminaries. Let $M$ be a von Neumann algebra and $\varphi$ be a faithful normal semifinite weight on $M$. We use the usual notations $\mathfrak{n}_{\varphi}=\left\{x \in M: \varphi\left(x^{*} x\right)<\infty\right\}$ and $m_{\varphi}=\mathfrak{n}_{\varphi}^{*} \mathfrak{n}_{\varphi}$. Let $\left(\mathcal{H}_{\varphi}, \pi_{\varphi}\right)$ be the GNS representation of $M$ induced by $\varphi$ where the canonical injection of $\mathfrak{n}_{\varphi}$ into $\mathcal{H}_{\varphi}$ is denoted by $x \mapsto x_{\varphi}$. Then $\mathfrak{A}_{\varphi}=\left\{x_{\varphi}\right.$ : $\left.x \in \mathfrak{n}_{\varphi} \cap \mathfrak{n}_{\varphi}^{*}\right\}$ is an achieved left Hilbert algebra and $\pi_{\varphi}(M)$ is its left von Neumann algebra. Let $\Delta_{\varphi}, J_{\varphi}$ and $\sigma_{t}^{\varphi}$ be the modular operator, the modular conjugation and the modular automorphism group, respectively, associated with $\varphi$.

Let $N$ be a unital von Neumann subalgebra of $M$. The conditional expectation $\varepsilon$ : $M \rightarrow N$ with respect to $\varphi$ is a unique norm one normal projection $\varepsilon$ of $M$ onto $N$ such that $\varphi(x)=\varphi(\varepsilon(x))$ for all $x \in M_{+}$. According to Takesaki [12], there exists the conditional expectation $\varepsilon: M \rightarrow N$ with respect to $\varphi$ if and only if $\varphi \uparrow N$ is semifinite and $\sigma_{t}^{\varphi}(N)=N$ for every $t \in \mathbf{R}$. Recently the concept of conditional expectations has been extended by Accardi and Cecchini [1]. Now suppose only that $\psi=\varphi \uparrow N$ is semifinite, and let $\left(\mathcal{H}_{\psi}, \pi_{\psi}\right)$ and $J_{\psi}$ be the GNS representation of $N$ and the modular conjugation associated with $\psi$. Since the canonical injection of $n_{\psi}=n_{\varphi}$ $\cap N$ into $\mathcal{H}_{\psi}$ is taken as the restriction of $x \mapsto x_{\varphi}$ to $\mathfrak{n}_{\psi}, \mathcal{H}_{\psi}$ is identified with a subspace of $\mathcal{H}_{\varphi}$. Let $P$ be the orthogonal projection of $\mathcal{H}_{\varphi}$ onto $\mathcal{H}_{\psi}$, then $P \in \pi_{\varphi}(N)^{\prime}$ and $\pi_{\psi}(x)=\pi_{\varphi}(x) P$ for all $x \in N$. The conditional expectation $\varepsilon: M \rightarrow N$ with respect to $\varphi$ is introduced in [1] by

$$
\pi_{\psi}(\varepsilon(x))=J_{\psi} P J_{\varphi} \pi_{\varphi}(x) J_{\varphi} J_{\psi}, \quad x \in M
$$

which is a faithful normal completely positive map of $M$ into $N$ such that $\varepsilon(1)=1$ and $\varphi(x)=\varphi(\varepsilon(x))$ for all $x \in M_{+}$(cf. [1, Theorem 7.5]). We here call $\varepsilon$ the generalized conditional expectation with respect to $\varphi$. This coincides with the conditional expectation (as a norm one projection onto $N$ ) with respect to $\varphi$ whenever the latter exists.

In the following lemmas, we assume that $\psi=\varphi \uparrow N$ is semifinite and $\varepsilon: M \rightarrow N$ is the generalized conditional expectation with respect to $\varphi$.

LEMMA'1. If $x \in \mathfrak{n}_{\varphi}$, then $\varepsilon(x) \in \mathfrak{n}_{\psi}$ and

$$
(\varepsilon(x))_{\varphi}=J_{\psi} P J_{\varphi} x_{\varphi} .
$$

Proof. Let $x \in n_{\varphi}$. Then $\varepsilon(x) \in n_{\psi}$ follows from

$$
\psi\left(\varepsilon(x)^{*} \varepsilon(x)\right) \leqslant \psi\left(\varepsilon\left(x^{*} x\right)\right)=\varphi\left(x^{*} x\right)<\infty .
$$


Choosing a net $\left\{a_{j}\right\}$ in $\mathrm{m}_{\psi} \cap N_{+}$with $a_{j} \nearrow 1$, we have

$$
\begin{aligned}
(\varepsilon(x))_{\varphi} & =\lim _{j} J_{\psi} \pi_{\psi}\left(a_{j}\right) J_{\psi}(\varepsilon(x))_{\varphi}=\lim _{j} \pi_{\psi}(\varepsilon(x)) J_{\psi}\left(a_{j}\right)_{\varphi} \\
& =\lim _{j} J_{\psi} P J_{\varphi} \pi_{\varphi}(x) J_{\varphi}\left(a_{j}\right)_{\varphi}=\lim _{j} J_{\psi} P \pi_{\varphi}\left(a_{j}\right) J_{\varphi} x_{\varphi} \\
& =J_{\psi} P J_{\varphi} x_{\varphi} . \quad \text { Q.E.D. }
\end{aligned}
$$

LEMMA 2. If $\gamma$ is a *-automorphism of $M$ and $\tilde{\varepsilon}: M \rightarrow \gamma^{-1}(N)$ is the generalized conditional expectation with respect to $\varphi \circ \gamma$, then $\tilde{\varepsilon}=\gamma^{-1} \circ \varepsilon \circ \gamma$.

Proof. Let $\tilde{\varphi}=\varphi \circ \gamma, \tilde{\psi}=\tilde{\varphi} \uparrow \gamma^{-1}(N)$ and $\tilde{P}$ be the orthogonal projection of $\mathcal{H}_{\tilde{\varphi}}$ onto $\mathcal{H}_{\tilde{\psi}}$. There is a unitary operator $U$ of $\mathcal{H}_{\tilde{\varphi}}$ onto $\mathcal{H}_{\varphi}$ such that $U x_{\tilde{\varphi}}=(\gamma(x))_{\varphi}$ for all $x \in \mathfrak{n}_{\tilde{\varphi}}$. Then it is readily seen that $P=U \tilde{P} U^{*}, J_{\varphi}=U J_{\tilde{\varphi}} U^{*}$ and $\pi_{\varphi}(\gamma(x))=$ $U \pi_{\tilde{\varphi}}(x) U^{*}$ for all $x \in M$. Hence

$$
\begin{aligned}
\pi_{\psi}(\gamma(\tilde{\varepsilon}(x))) & =U \pi_{\tilde{\psi}}(\tilde{\varepsilon}(x)) U^{*}=U J_{\tilde{\psi}} \tilde{P} J_{\tilde{\varphi}} \pi_{\tilde{\varphi}}(x) J_{\tilde{\varphi}} J_{\tilde{\psi}} U^{*} \\
& =J_{\psi} P J_{\varphi} \pi_{\varphi}(\gamma(x)) J_{\varphi} J_{\psi}=\pi_{\psi}(\varepsilon(\gamma(x))), \quad x \in M,
\end{aligned}
$$

so that we have $\gamma \circ \tilde{\varepsilon}=\varepsilon \circ \gamma$. Q.E.D.

2. Increasing case. In this section we discuss the martingale convergence of generalized conditional expectations for the increasing case. We fix an increasing net $\left\{N_{\alpha}\right\}$ of unital von Neumann subalgebras of $M$ and let $N_{\infty}=V_{\alpha} N_{\alpha}$. Let $\varphi$ be a faithful normal semifinite weight on $M$. Assume that $\psi_{\alpha}=\varphi \uparrow N_{\alpha}$ is semifinite for each $\alpha$ and hence also $\psi_{\infty}=\varphi \uparrow N_{\infty}$ is semifinite. For each $\alpha$, we take $\mathfrak{n}_{\alpha}=\mathfrak{n}_{\varphi} \cap N_{\alpha}$, $\mathfrak{m}_{\alpha}=\mathfrak{n}_{\alpha}^{*} \mathfrak{n}_{\alpha}$, the GNS representation $\left(\mathcal{H}_{\alpha}, \pi_{\alpha}\right)$ of $N_{\alpha}$, the left Hilbert algebra $\mathfrak{A}_{\alpha}=\left\{x_{\varphi}: x \in \mathfrak{n}_{\alpha} \cap \mathfrak{n}_{\alpha}^{*}\right\}$, the modular operator $\Delta_{\alpha}$, the modular conjugation $J_{\alpha}$ and the modular automorphism group $\sigma_{t}^{\alpha}$ associated with $\psi_{\alpha}$. then $\left\{\mathcal{H}_{\alpha}\right\}$ is an increasing net of subspaces of $\mathcal{K}_{\varphi}$. Let $P_{\alpha}$ be the orthogonal projection of $\mathcal{H}_{\varphi}$ onto $\mathcal{H}_{\alpha}$. We further take $\mathfrak{n}_{\infty}, \mathfrak{m}_{\infty},\left(\mathcal{H}_{\infty}, \pi_{\infty}\right), \mathfrak{A}_{\infty}, \Delta_{\infty}, J_{\infty}, \sigma_{t}^{\infty}$ and $P_{\infty}$ analogously associated with $\psi_{\infty}$. The generalized conditional expectations $\varepsilon_{\alpha}: M \rightarrow N_{\alpha}$ and $\varepsilon_{\infty}: M \rightarrow N_{\infty}$ with respect to $\varphi$ are given as follows:

$$
\begin{aligned}
\pi_{\alpha}\left(\varepsilon_{\alpha}(x)\right) & =J_{\alpha} P_{\alpha} J_{\varphi} \pi_{\varphi}(x) J_{\varphi} J_{\alpha}, \\
\pi_{\infty}\left(\varepsilon_{\infty}(x)\right) & =J_{\infty} P_{\infty} J_{\varphi} \pi_{\varphi}(x) J_{\varphi} J_{\infty}, \quad x \in M .
\end{aligned}
$$

Under the above assumptions and notations, we now state

THEOREM 3. The following conditions are equivalent:

(i) $\cup_{\alpha} \mathfrak{A}_{\alpha}\left(\subset \mathfrak{A}_{\infty}\right)$ is a core of $\Delta_{\infty}^{1 / 2}$;

(ii) $\left\|\left(\varepsilon_{\alpha}(x)\right)_{\varphi}-\left(\varepsilon_{\infty}(x)\right)_{\varphi}\right\| \rightarrow 0$ for every $x \in \mathrm{n}_{\varphi}$;

(iii) $s$ - $\lim _{\alpha} \varepsilon_{\alpha}(x)=\varepsilon_{\infty}(x)$ for every $x \in M$;

(iv) $\left\|f \circ \varepsilon_{\alpha}-f \circ \varepsilon_{\infty}\right\| \rightarrow 0$ for every $f \in M_{*}$.

If $\varphi$ is bounded (i.e., $\varphi(1)<\infty$ ), then the above conditions (i)-(iv) are fulfilled.

Proof. (i) $\Rightarrow$ (ii). If $x \in \mathfrak{n}_{\varphi}$, then by Lemma 1 we have

$$
\left(\varepsilon_{\alpha}(x)\right)_{\varphi}=J_{\alpha} P_{\alpha} J_{\varphi} x_{\varphi}, \quad\left(\varepsilon_{\infty}(x)\right)_{\varphi}=J_{\infty} P_{\infty} J_{\varphi} x_{\varphi} .
$$


Therefore the condition (ii) is equivalent to $s-\lim _{\alpha} J_{\alpha} P_{\alpha}=J_{\infty} P_{\infty}$, and so we may assume $N_{\infty}=M$ and show $s-\lim _{\alpha} J_{\alpha} P_{\alpha}=J_{\varphi}$. Let $f \in M_{*}^{+}$with $f \leqslant \varphi$ be given. By [16, Theorem 3.2], there exists a unique $h \in \mathrm{m}_{\varphi}$ with $0 \leqslant h \leqslant 1$ such that

$$
f(x)=\frac{1}{2} \varphi(h x+x h), \quad x \in \mathfrak{n}_{\varphi} \cap \mathfrak{n}_{\varphi}^{*} .
$$

For each $\alpha$, there further exists a unique $h_{\alpha} \in \mathfrak{m}_{\alpha}$ with $0 \leqslant h_{\alpha} \leqslant 1$ such that

$$
f(x)=\frac{1}{2} \varphi\left(h_{\alpha} x+x h_{\alpha}\right), \quad x \in \mathfrak{n}_{\alpha} \cap \mathfrak{n}_{\alpha}^{*} .
$$

Under the assumption (i), the following results (1)-(3) have been proved in [8].

(1) $h_{\varphi} \in D\left(\Delta_{\varphi}\right),\left(h_{\alpha}\right)_{\varphi} \in D\left(\Delta_{\alpha}\right),\left\|\left(h_{\alpha}\right)_{\varphi}-h_{\varphi}\right\| \rightarrow 0$ and $\left\|\Delta_{\alpha}\left(h_{\alpha}\right)_{\varphi}-\Delta_{\varphi} h_{\varphi}\right\| \rightarrow 0$.

(2) The set $\left\{\left(1+\Delta_{\varphi}\right) h_{\varphi}\right\}$ is total in $\mathcal{H}_{\varphi}$ where $h \in \mathrm{m}_{\varphi}$ with $0 \leqslant h \leqslant 1$ is taken as above for any $f \in M_{*}^{+}$with $f \leqslant \varphi$.

(3) Let $\tilde{\Delta}_{\alpha}=\Delta_{\alpha} P_{\alpha}+\left(1-P_{\alpha}\right)$. Then $s$-lim $\tilde{\Delta}_{\alpha} \tilde{\Delta}_{\alpha}^{i t}=\Delta_{\varphi}^{i t}$ uniformly for $t$ in any finite interval.

We now define vector-valued continuous functions $F_{\alpha}(z)$ on the strip $0 \leqslant \operatorname{Re} z \leqslant 1$, analytic in the interior, by

$$
F_{\alpha}(z)=e^{z^{2}}\left(\Delta_{\alpha}^{z}\left(h_{\alpha}\right)_{\varphi}-\Delta_{\varphi}^{z} h_{\varphi}\right), \quad 0 \leqslant \operatorname{Re} z \leqslant 1
$$

Since

$$
\begin{gathered}
\left\|F_{\alpha}(i t)\right\| \leqslant e^{-t^{2}}\left\|\left(h_{\alpha}\right)_{\varphi}-h_{\varphi}\right\|+e^{-t^{2}}\left\|\left(\tilde{\Delta}_{\alpha}^{i t}-\Delta_{\varphi}^{i t}\right) h_{\varphi}\right\|, \\
\left\|F_{\alpha}(1+i t)\right\| \leqslant e^{1-t^{2}}\left\|\Delta_{\alpha}\left(h_{\alpha}\right)_{\varphi}-\Delta_{\varphi} h_{\varphi}\right\|+e^{1-t^{2}}\left\|\left(\tilde{\Delta}_{\alpha}^{i t}-\Delta_{\varphi}^{i t}\right) \Delta_{\varphi} h_{\varphi}\right\|, \quad t \in \mathbf{R},
\end{gathered}
$$

we have

$$
\begin{aligned}
e^{1 / 4}\left\|J_{\alpha}\left(h_{\alpha}\right)_{\varphi}-J_{\varphi} h_{\varphi}\right\| & =e^{1 / 4}\left\|\Delta_{\alpha}^{1 / 2}\left(h_{\alpha}\right)_{\varphi}-\Delta_{\varphi}^{1 / 2} h_{\varphi}\right\|=\left\|F_{\alpha}(1 / 2)\right\| \\
& \leqslant \sup _{t \in \mathbf{R}}\left(\left\|F_{\alpha}(i t)\right\|,\left\|F_{\alpha}(1+i t)\right\|\right) \rightarrow 0
\end{aligned}
$$

by using (1) and (3). Hence

$$
\left\|J_{\alpha} P_{\alpha} h_{\varphi}-J_{\varphi} h_{\varphi}\right\| \leqslant\left\|\left(h_{\alpha}\right)_{\varphi}-h_{\varphi}\right\|+\left\|J_{\alpha}\left(h_{\alpha}\right)_{\varphi}-J_{\varphi} h_{\varphi}\right\| \rightarrow 0 .
$$

Moreover, the set $\left\{h_{\varphi}\right\}$, taken for any $f \in M_{*}^{+}$with $f \leqslant \varphi$, is total in $\mathcal{H}_{\varphi}$, because (2) implies

$$
\left\{h_{\varphi}\right\}^{\perp}=\left(\left(1+\Delta_{\varphi}\right)^{-1} \mathcal{H}_{\varphi}\right)^{\perp}=\{0\}
$$

Thus $s-\lim _{\alpha} J_{\alpha} P_{\alpha}=J_{\varphi}$ is proved.

(ii) $\Rightarrow$ (i). Suppose that $s$ - $\lim _{\alpha} J_{\alpha} P_{\alpha}=J_{\infty} P_{\infty}$ and hence $s$ - $\lim _{\alpha} J_{\alpha} P_{\alpha} J_{\infty} P_{\infty}=P_{\infty}$. Take the generalized conditional expectation $\varepsilon_{\infty, \alpha}: N_{\infty} \rightarrow N_{\alpha}$ with respect to $\psi_{\infty}$. Given $x \in \mathfrak{n}_{\infty} \cap \mathfrak{n}_{\infty}^{*}$, by Lemma 1 we obtain, for each $\alpha, \varepsilon_{\infty, \alpha}(x) \in \mathrm{n}_{\alpha} \cap \mathrm{n}_{\alpha}^{*}$ and

$$
\begin{gathered}
\left\|\left(\varepsilon_{\infty, \alpha}(x)\right)_{\varphi}-x_{\varphi}\right\|=\left\|J_{\alpha} P_{\alpha} J_{\infty} x_{\varphi}-x_{\varphi}\right\| \rightarrow 0, \\
\left\|\Delta_{\infty}^{1 / 2}\left(\varepsilon_{\infty, \alpha}(x)\right)_{\varphi}-\Delta_{\infty}^{1 / 2} x_{\varphi}\right\|=\left\|\left(\varepsilon_{\infty, \alpha}\left(x^{*}\right)\right)_{\varphi}-\left(x^{*}\right)_{\varphi}\right\|=\left\|J_{\alpha} P_{\alpha} J_{\infty}\left(x^{*}\right)_{\varphi}-\left(x^{*}\right)_{\varphi}\right\| \rightarrow 0 .
\end{gathered}
$$

Thus (i) is satisfied. 
(i) $\Rightarrow$ (iii). Given $x \in M$ and $\xi \in \mathcal{H}_{\infty}$, we have

$$
\begin{aligned}
& \left\|\pi_{\infty}\left(\varepsilon_{\alpha}(x)\right) \xi-\pi_{\infty}\left(\varepsilon_{\infty}(x)\right) \xi\right\| \\
& \quad \leqslant\left\|\pi_{\infty}\left(\varepsilon_{\alpha}(x)\right)\left(P_{\alpha} \xi-\xi\right)\right\|+\left\|\pi_{\alpha}\left(\varepsilon_{\alpha}(x)\right) P_{\alpha} \xi-\pi_{\infty}\left(\varepsilon_{\infty}(x)\right) \xi\right\| \\
& \quad \leqslant\|x\|\left\|P_{\alpha} \xi-\xi\right\|+\left\|J_{\alpha} P_{\alpha} J_{\varphi} \pi_{\varphi}(x) J_{\varphi} J_{\alpha} P_{\alpha} \xi-J_{\infty} P_{\infty} J_{\varphi} \pi_{\varphi}(x) J_{\varphi} J_{\infty} \xi\right\| \\
& \quad \rightarrow 0,
\end{aligned}
$$

because $s-\lim _{\alpha} J_{\alpha} P_{\alpha}=J_{\infty} P_{\infty}$ and hence $s-\lim _{\alpha} P_{\alpha}=P_{\infty}$. Thus $s-\lim _{\alpha} \varepsilon_{\alpha}(x)=\varepsilon_{\infty}(x)$.

(i) $\Rightarrow$ (iv). Since $\pi_{\infty}\left(N_{\infty}\right)$ on $\mathcal{H}_{\infty}$ is a standard form of $N_{\infty}$, given $f \in M_{*}^{+}$there is a $\xi \in \mathcal{H}_{\infty}$ such that $f(x)=\left\langle\pi_{\infty}(x) \xi, \xi\right\rangle$ for all $x \in N_{\infty}$ (cf. [7, Lemma 2.10]). For every $x \in M$, we have

$$
\begin{aligned}
& \left|f\left(\varepsilon_{\alpha}(x)\right)-f\left(\varepsilon_{\infty}(x)\right)\right| \leqslant\left|\left\langle\pi_{\infty}\left(\varepsilon_{\alpha}(x)\right)\left(\xi-P_{\alpha} \xi\right), \xi\right\rangle\right| \\
& +\left|\left\langle\pi_{\alpha}\left(\varepsilon_{\alpha}(x)\right) P_{\alpha} \xi, \xi\right\rangle-\left\langle\pi_{\infty}\left(\varepsilon_{\infty}(x)\right) \xi, \xi\right\rangle\right| \\
& \leqslant\|x\|\left\|P_{\alpha} \xi-\xi\right\|\|\xi\| \\
& +\left|\left\langle\pi_{\varphi}(x) J_{\varphi} J_{\alpha} P_{\alpha} \xi, J_{\varphi} J_{\alpha} P_{\alpha} \xi\right\rangle-\left\langle\pi_{\varphi}(x) J_{\varphi} J_{\infty} P_{\infty} \xi, J_{\varphi} J_{\infty} P_{\infty} \xi\right\rangle\right| \\
& \leqslant\|x\|\|\xi\|\left(\left\|P_{\alpha} \xi-\xi\right\|+2\left\|J_{\alpha} P_{\alpha} \xi-J_{\infty} P_{\infty} \xi\right\|\right) \text {. }
\end{aligned}
$$

This shows that (i) implies (iv).

(iii) $\Rightarrow$ (ii) and (iv) $\Rightarrow$ (ii). If either (iii) or (iv) holds, then $w$ - $\lim _{\alpha} \varepsilon_{\alpha}(x)=\varepsilon_{\infty}(x)$ for all $x \in M$. For every $x \in \mathfrak{n}_{\varphi}$, noting that $\left\{\left(\varepsilon_{\alpha}(x)\right)_{\varphi}\right\}$ is bounded in $\mathcal{H}_{\infty}$, let $\xi$ be any weak accumulation point of $\left\{\left(\varepsilon_{\alpha}(x)\right)_{\varphi}\right\}$ and choose a subnet $\left\{\left(\varepsilon_{\alpha^{\prime}}(x)\right)_{\varphi}\right\}$ of $\left\{\left(\varepsilon_{\alpha}(x)\right)_{\varphi}\right\}$ such that $\left(\varepsilon_{\alpha^{\prime}}(x)\right)_{\varphi} \rightarrow \xi$ weakly. Since $w$-lim $\lim _{\alpha^{\prime}} \varepsilon_{\alpha^{\prime}}(x)=\varepsilon_{\infty}(x)$, we then have $\xi=$ $\left(\varepsilon_{\infty}(x)\right)_{\varphi}$ (cf. [10, p. 28]). Hence it follows that $\left(\varepsilon_{\alpha}(x)\right)_{\varphi} \rightarrow\left(\varepsilon_{\infty}(x)\right)_{\varphi}$ weakly. On the other hand, $\left\|\left(\varepsilon_{\alpha}(x)\right)_{\varphi}\right\| \leqslant\left\|\left(\varepsilon_{\infty}(x)\right)_{\varphi}\right\|$ holds for each $\alpha$ by Lemma 1. Thus $\|\left(\varepsilon_{\alpha}(x)\right)_{\varphi}-$ $\left(\varepsilon_{\infty}(x)\right)_{\varphi} \| \rightarrow 0$.

Finally let $\varphi$ be bounded. For every $x \in N_{\infty}$, there is a net $\left\{x_{j}\right\}$ in $\cup_{\alpha} N_{\alpha}$ such that $s-\lim _{j} x_{j}=x$ and $s-\lim _{j} x_{j}^{*}=x^{*}$. We then have $\left\|\left(x_{j}\right)_{\varphi}-x_{\varphi}\right\| \rightarrow 0$ and $\| \Delta_{\infty}^{1 / 2}\left(x_{j}\right)_{\varphi}-$ $\Delta_{\infty}^{1 / 2} x_{\varphi}\|=\|\left(x_{j}^{*}\right)_{\varphi}-\left(x^{*}\right)_{\varphi} \| \rightarrow 0$. Hence the condition (i) is satisfied. Q.E.D.

ReMARKS. (1) When each $\varepsilon_{\alpha}$ is the conditional expectation (as a norm one projection) with respect to $\varphi$, the martingale convergence properties (iii) and (iv) are satisfied (see [13]).

(2) Under the condition (i), it can be proved (see [8]) that $s-\lim _{\alpha} \sigma_{t}^{\alpha}(x)=\sigma_{t}^{\infty}(x)$ uniformly for $t$ in any finite interval for every $x \in \cup_{\alpha} N_{\alpha}$. In this case, it further holds that $s$ - $\lim _{\alpha} \sigma_{t}^{\alpha}\left(\varepsilon_{\alpha}(x)\right)=\sigma_{t}^{\infty}\left(\varepsilon_{\infty}(x)\right)$ uniformly for $t$ in any finite interval for every $x \in M$.

3. Decreasing case. In this section we deal with the convergence of generalized conditional expectations for the decreasing case. Let $\left\{N_{\alpha}\right\}$ be a decreasing net of unital von Neumann subalgebras of $M$ with $N_{\infty}=\bigcap_{\alpha} N_{\alpha}$. Let $\varphi$ be a faithful normal semifinite weight on $M$ such that $\psi_{\infty}=\varphi \mid N_{\infty}$ is semifinite and hence each $\psi_{\alpha}=\varphi \mid$ $N_{\alpha}$ is semifinite. We use the notations $\mathfrak{n}_{\alpha}, \mathfrak{m}_{\alpha},\left(\mathcal{H}_{\alpha}, \pi_{\alpha}\right), \Delta_{\alpha}, J_{\alpha}, P_{\alpha}$ and $\mathfrak{n}_{\infty}, \mathfrak{m}_{\infty}$, $\left(\mathcal{H}_{\infty}, \pi_{\infty}\right), \Delta_{\infty}, J_{\infty}, P_{\infty}$ as in $\S 2$. We take the generalized conditional expectations $\varepsilon_{\alpha}$ : $M \rightarrow N_{\alpha}$ and $\varepsilon_{\infty}: M \rightarrow N_{\infty}$ with respect to $\varphi$. 
THEOREM 4. Under the above assumptions, consider the following conditions:

(i) $s$ - $\lim _{\alpha} P_{\alpha}=P_{\infty}$ (i.e., $\cap_{\alpha} \mathcal{H}_{\alpha}=\mathcal{H}_{\infty}$ );

(ii) $\left\|\left(\varepsilon_{\alpha}(x)\right)_{\varphi}-\left(\varepsilon_{\infty}(x)\right)_{\varphi}\right\| \rightarrow 0$ for every $x \in \mathfrak{n}_{\varphi}$;

(iii) $s-\lim _{\alpha} \varepsilon_{\alpha}(x)=\varepsilon_{\infty}(x)$ for every $x \in M$;

(iv) $\left\|f \circ \varepsilon_{\alpha}-f \circ \varepsilon_{\infty}\right\| \rightarrow 0$ for every $f \in M_{*}$.

Then the conditions (i) and (ii) are equivalent and the condition (i) implies (iii) and (iv). If $\varphi$ is bounded, then the conditions (i)-(iii) are equivalent.

Proof. (i) $\Rightarrow$ (ii). By Lemma 1 , the condition (ii) is equivalent to $s-\lim _{\alpha} J_{\alpha} P_{\alpha}=$ $J_{\infty} P_{\infty}$. For each $f \in M_{*}^{+}$with $f \leqslant \varphi$, there exist, by [16, Theorem 3.2], unique $h_{\alpha} \in \mathfrak{m}_{\alpha}$ and $h_{\infty} \in \mathfrak{m}_{\infty}$ with $0 \leqslant h_{\alpha} \leqslant 1,0 \leqslant h_{\infty} \leqslant 1$ such that

$$
\begin{array}{cc}
f(x)=\frac{1}{2} \varphi\left(h_{\alpha} x+x h_{\alpha}\right), & x \in \mathfrak{n}_{\alpha} \cap \mathfrak{n}_{\alpha}^{*}, \\
f(x)=\frac{1}{2} \varphi\left(h_{\infty} x+x h_{\infty}\right), & x \in \mathfrak{n}_{\infty} \cap \mathfrak{n}_{\infty}^{*} .
\end{array}
$$

Under the assumption (i), the following results (1)-(3) hold (see [8]).

(1) $\left(h_{\alpha}\right)_{\varphi} \in D\left(\Delta_{\alpha}\right), \quad\left(h_{\infty}\right)_{\varphi} \in D\left(\Delta_{\infty}\right),\left\|\left(h_{\alpha}\right)_{\varphi}-\left(h_{\infty}\right)_{\varphi}\right\| \rightarrow 0$ and $\| \Delta_{\alpha}\left(h_{\alpha}\right)_{\varphi}-$ $\Delta_{\infty}\left(h_{\infty}\right)_{\phi} \| \rightarrow 0$.

(2) The set $\left\{\left(1+\Delta_{\infty}\right)\left(h_{\infty}\right)_{\varphi}\right\}$ is total in $\mathcal{H}_{\infty}$ where $h_{\infty} \in \mathfrak{m}_{\infty}$ with $0 \leqslant h_{\infty} \leqslant 1$ is taken as above for any $f \in M_{*}^{+}$with $f \leqslant \varphi$.

(3) Let $\tilde{\Delta}_{\alpha}=\Delta_{\alpha} P_{\alpha}+\left(1-P_{\alpha}\right)$ and $\tilde{\Delta}_{\infty}=\Delta_{\infty} P_{\infty}+\left(1-P_{\infty}\right)$. Then $s-\lim _{\alpha} \tilde{\Delta}_{\alpha}^{i t}=$ $\tilde{\Delta}_{\infty}^{i t}$ uniformly for $t$ in any finite interval.

Defining

$$
F_{\alpha}(z)=e^{z^{2}}\left(\Delta_{\alpha}^{z}\left(h_{\alpha}\right)_{\varphi}-\Delta_{\infty}^{z}\left(h_{\infty}\right)_{\varphi}\right), \quad 0 \leqslant \operatorname{Re} z \leqslant 1,
$$

and using (1) and (3), we have

$$
\left\|F_{\alpha}\left(\frac{1}{2}\right)\right\|=e^{1 / 4}\left\|J_{\alpha}\left(h_{\alpha}\right)_{\varphi}-J_{\infty}\left(h_{\infty}\right)_{\varphi}\right\| \rightarrow 0
$$

as in the proof of Theorem 3 . Hence

$$
\left\|J_{\alpha}\left(h_{\infty}\right)_{\varphi}-J_{\infty}\left(h_{\infty}\right)_{\varphi}\right\| \leqslant\left\|\left(h_{\infty}\right)_{\varphi}-\left(h_{\alpha}\right)_{\varphi}\right\|+\left\|J_{\alpha}\left(h_{\alpha}\right)_{\varphi}-J_{\infty}\left(h_{\infty}\right)_{\varphi}\right\| \rightarrow 0 .
$$

Since (2) shows that the set $\left\{\left(h_{\infty}\right)_{\varphi}\right\}$ is total in $\mathcal{H}_{\infty}, J_{\alpha} P_{\alpha}=J_{\alpha} P_{\infty}+J_{\alpha}\left(P_{\alpha}-P_{\infty}\right)$ converges strongly to $J_{\infty} P_{\infty}$.

(ii) $\Rightarrow$ (i). If $s-\lim _{\alpha} J_{\alpha} P_{\alpha}=J_{\infty} P_{\infty}$, then for any $\xi \in \cap_{\alpha} \mathcal{H}_{\alpha}$ we have

$$
\left\|P_{\infty} \xi\right\|=\left\|J_{\infty} P_{\infty} \xi\right\|=\lim _{\alpha}\left\|J_{\alpha} P_{\alpha} \xi\right\|=\|\xi\|,
$$

so that $\xi \in \mathcal{H}_{\infty}$. Hence (i) holds.

(i) $\Rightarrow$ (iii). Suppose that $s-\lim _{\alpha} J_{\alpha} P_{\alpha}=J_{\infty} P_{\infty}$. Given $x \in M$ and $\xi \in \mathcal{H}_{\infty}$, we have

$\left\|\pi_{\varphi}\left(\varepsilon_{\alpha}(x)\right) \xi-\pi_{\varphi}\left(\varepsilon_{\infty}(x)\right) \xi\right\|=\left\|J_{\alpha} P_{\alpha} J_{\varphi} \pi_{\varphi}(x) J_{\varphi} J_{\alpha} P_{\alpha} \xi-J_{\infty} P_{\infty} J_{\varphi} \pi_{\varphi}(x) J_{\varphi} J_{\infty} P_{\infty} \xi\right\| \rightarrow 0$.

Choosing a net $\left\{a_{j}\right\}$ in $\mathfrak{m}_{\infty} \cap\left(N_{\infty}\right)_{+}$with $a_{j} \nearrow 1$, we have

$$
J_{\varphi} x_{\varphi}=\lim _{j} \pi_{\varphi}\left(a_{j}\right) J_{\varphi} x_{\varphi}=\lim _{j} J_{\varphi} \pi_{\varphi}(x) J_{\varphi}\left(a_{j}\right)_{\varphi}, \quad x \in \mathrm{n}_{\varphi} .
$$

This shows that $\pi_{\varphi}(M)^{\prime} \mathcal{H}_{\infty}$ is dense in $\mathcal{H}_{\varphi}$. Hence (iii) holds. 
(i) $\Rightarrow$ (iv). Since $\pi_{\varphi}(M)^{\prime} \mathcal{H}_{\infty}$ is dense in $\mathcal{H}_{\varphi}$, it suffices to prove (iv) for $f \in M_{*}^{+}$ such that

$$
f(x)=\left\langle\pi_{\varphi}(x) a^{\prime} \xi, a^{\prime} \xi\right\rangle, \quad x \in M,
$$

where $a^{\prime} \in \pi_{\varphi}(M)^{\prime}$ and $\xi \in \mathcal{H}_{\infty}^{\prime}$. For this $f$, we have

$$
\begin{aligned}
& \left|f\left(\varepsilon_{\alpha}(x)\right)-f\left(\varepsilon_{\infty}(x)\right)\right|=\left|\left\langle\pi_{\varphi}\left(\varepsilon_{\alpha}(x)\right) \xi, a^{\prime *} a^{\prime} \xi\right\rangle-\left\langle\pi_{\varphi}\left(\varepsilon_{\infty}(x)\right) \xi, a^{\prime *} a^{\prime} \xi\right\rangle\right| \\
& \quad=\left|\left\langle\pi_{\varphi}(x) J_{\varphi} J_{\alpha} \xi, J_{\varphi} J_{\alpha} P_{\alpha} a^{\prime *} a^{\prime} \xi\right\rangle-\left\langle\pi_{\varphi}(x) J_{\varphi} J_{\infty} \xi, J_{\varphi} J_{\infty} P_{\infty} a^{\prime *} a^{\prime} \xi\right\rangle\right| \\
& \quad \leqslant\|x\|\left(\left\|J_{\alpha} \xi-J_{\infty} \xi\right\|\left\|a^{\prime *} a^{\prime} \xi\right\|+\|\xi\|\left\|\left(J_{\alpha} P_{\alpha}-J_{\infty} P_{\infty}\right) a^{\prime *} a^{\prime} \xi\right\|\right) .
\end{aligned}
$$

If (i) is satisfied, then it follows from the proof of (i) $\Rightarrow$ (ii) that $\left\|J_{\alpha} \xi-J_{\infty} \xi\right\| \rightarrow 0$ for all $\xi \in \mathcal{K}_{x}$. Therefore $\left\|f \circ \varepsilon_{\alpha}-f \circ \varepsilon_{x}\right\| \rightarrow 0$.

Finally if $\varphi$ is bounded, then it is clear that (iii) implies (ii). Q.E.D.

REMARKS. (1) If each $\varepsilon_{\alpha}$ is the conditional expectation (as a norm one projection) with respect to $\varphi$, then the martingale convergence properties (iii) and (iv) hold (see [13]). In this case, $\left(\varepsilon_{\alpha}(x)\right)_{\varphi}=P_{\alpha} x_{\varphi}$ and $\left(\varepsilon_{x}(x)\right)_{\varphi}=P_{x} x_{\varphi}$ for all $x \in n_{\varphi}$. Hence the conditions (i) and (ii) are also satisfied (see the proof of (iii) $\Rightarrow$ (ii) in Theorem 3 ).

(2) We can find a von Neumann algebra $M$ with a cyclic and separating vector $\xi$ and a decreasing net $\left\{N_{\alpha}\right\}$ of von Neumann subalgebras of $M$ such that $\xi$ is cyclic for each $N_{\alpha}$ and $N_{\infty}=\bigcap_{\alpha} N_{\alpha}=\mathrm{Cl}$ (cf. [3. Example 1]). Let $\varphi(x)=\langle x \xi, \xi\rangle$ for $x \in M$, then $\mathfrak{H}_{\alpha}=\mathfrak{K}_{\varphi}$ for each $\alpha$ and $\mathfrak{H}_{x}=\mathbf{C} \xi$. Thus the conditions (i)-(iii) in Theorem 4 are not satisfied for this case.

(3) Under the condition (i), it follows (see [8]) that $s-\lim _{\alpha} \sigma_{t}^{\alpha}(x)=\sigma_{t}^{\infty}(x)$ uniformly for $t$ in any finite interval for every $x \in M_{\infty}$, and further $s$-lim ${ }_{\alpha} \sigma_{t}^{\alpha}\left(\varepsilon_{\alpha}(x)\right)=$ $\sigma_{t}^{\infty}\left(\varepsilon_{\infty}(x)\right)$ uniformly for $t$ in any finite interval for every $x \in M$.

As an example we lastly consider nonabelian $K$-flows studied by Emch [5,6]. A nonabelian $K$-flow is described by $\left(M, \varphi, \gamma_{t}, N_{0}\right)$ where $M$ is a von Neumann algebra, $\varphi$ is a faithful normal state on $M, \gamma_{t}$ is a one-parameter automorphism group of $M$, and $N_{0}$ is a von Neumann subalgebra of $M$ such that $\varphi$ is $\gamma_{t}$-invariant and

$$
\begin{aligned}
& N_{0} \subset \gamma_{t}\left(N_{0}\right), \quad t \geqslant 0, \\
& \bigcap_{t \in \mathbf{R}} \overline{\gamma_{t}\left(N_{0}\right) \xi}=\mathbf{C} \xi, \quad \bigvee_{t \in \mathbf{R}} \gamma_{t}\left(N_{0}\right)=M \text {. }
\end{aligned}
$$

Here it is not necessarily assumed that $\sigma_{t}{ }^{\varphi}\left(N_{0}\right)=N_{0}$ for all $t \in \mathbf{R}$. Let $N_{t}=\gamma_{t}\left(N_{0}\right)$ and $\varepsilon_{t}: M \rightarrow N_{t}$ be the generalized conditional expectation with respect to $\varphi$. Then $\left\{N_{t}: t \in \mathbf{R}\right\}$ is an increasing net of unital von Neumann subalgebras of $M$ with $V_{t} N_{t}=M$ and $\cap_{t} N_{t}=\mathrm{Cl}$, and the condition (i) in Theorem 3 (resp. Theorem 4) is satisfied for $t \rightarrow \infty$ (resp. $t \rightarrow-\infty$ ). Moreover, it follows from Lemma 2 that $\varepsilon_{t}=\gamma_{t} \circ \varepsilon_{0} \circ \gamma_{-t}$ for all $t \in \mathbf{R}$. Therefore we conclude by Theorems 3 and 4 that for every $x \in M$ and $f \in M_{*}$,

$$
\begin{aligned}
& \lim _{t \rightarrow \infty} \varepsilon_{t}(x)=x, \quad \lim _{t \rightarrow-\infty} \varepsilon_{t}(x)=\varepsilon_{-\infty}(x)=\varphi(x) 1, \\
& \left\|f \circ \gamma_{t} \circ \varepsilon_{0}-f \circ \gamma_{t}\right\|=\left\|f \circ \varepsilon_{t}-f\right\| \rightarrow 0 \quad(\text { as } t \rightarrow \infty), \\
& \left\|f \circ \gamma_{t} \circ \varepsilon_{0}-f(1) \varphi\right\|=\left\|f \circ \varepsilon_{t}-f \circ \varepsilon_{-\infty}\right\| \rightarrow 0 \quad(\text { as } t \rightarrow-\infty) \text {. }
\end{aligned}
$$




\section{REFERENCES}

1. L. Accardi and C. Cecchini, Conditional expectations in von Neumann algebras and a theorem of Takesaki, J. Funct. Anal. 45 (1982), 245-273.

2. H. Araki, On the equivalence of the KMS condition and the variational principle for quantum lattice systems, Comm. Math. Phys. 38 (1974), 1-10.

3. H. Araki and A. Kishimoto, On clustering property, Rep. Math. Phys. 10 (1976), 275-281.

4. N. Dang-Ngoc, Pointwise convergence of martingales in von Neumann algehras, Israel J. Math. 34 (1979), 273-280.

5. G. G. Emch, Nonabelian special K-flows, J. Funct. Anal. 19 (1975), 1-12.

6. - Generalized K-flows, Comm. Math. Phys. 49 (1976), 191-215.

7. U. Haagerup, The standard form of von Neumann algebras, Math. Scand. 37 (1975), 271-283.

8. F. Hiai, Martingale type convergence of modular automorphism groups on von Neumann algebras, preprint 1982.

9. E. C. Lance, Martingale convergence in von Neumann algebras, Math. Proc. Cambridge Philos. Soc. 84 (1978), 47-56.

10. Ş. Strătilă, Modular theory in operator algebras, Editura Academiei \& Abacus Press, Tunbridge Wells, 1981 .

11. M. Takesaki, Tomita's theory of modular hilbert algebras and its applications, Lecture Notes in Math., vol. 128, Springer-Verlag, Berlin and New York, 1970.

12. Conditional expectations in von Neumann algebras, J. Funct. Anal. 9 (1972), 306-321.

13. M. Tsukada, Strong convergence of martingales in von Neumann algebras, Proc. Amer. Math. Soc. 88 (1983), 537-540.

14. H. Umegaki, Conditional expectation in an operator algebra, Tôhoku Math. J. 6 (1954), 177-181.

15. Conditional expectation in an operator algebra, II, Tôhoku Math. J. 8 (1956), 86-100.

16. A. Van Daele, A Radon Nikodym theorem for weights on von Neumann algebras, Pacific J. Math. 61 (1975), 527-542.

Department of Information Sciences, Science University of Tokyo, Noda City, Chiba 278, JAPAN 